\title{
On obtaining effective elasticity tensors with entries zeroing method
}

\author{
Bartosz Gierlach, Tomasz Danek \\ AGH University of Science and Technology, Faculty of Geology, Geophysics and Environmental Protection, \\ Department of Geoinformatics and Applied Computer Sciences; al. A. Mickiewicza 30, 30-059 Krakow, Poland; \\ e-mail: gierlach@agh.edu.pl,tdanek@agh.edu.pl
}

\begin{abstract}
(c) 2018 Authors. This is an open access publication, which can be used, distributed and reproduced in any medium according
\end{abstract} to the Creative Commons CC-BY 4.0 License requiring that the original work has been properly cited.

Received: 21 August 2017; accepted: 25 April 2018

\begin{abstract}
The purpose of this paper is to propose a new method for obtaining tensors expressing certain symmetries, called effective elasticity tensors, and their optimal orientation. The generally anisotropic tensor being the result of in situ seismic measurements describes the elastic properties of a medium. It can be approximated with a tensor of a specific symmetry class. With a known symmetry class and orientation, one can better describe geological structure elements like layers and fissures. A method used to obtain effective tensor in the previous papers (i.e. Danek \& Slawinski 2015) is based on minimizing the Frobenius norm between the measured and effective tensor of a chosen symmetry class in the same coordinate system. In this paper, we propose a new approach for obtaining the effective tensor with the assumption of a certain symmetry class. The entry zeroing method assumes the minimization of the target function, being the measure of similarity with the form of the effective tensor for the specific class. The optimization of orientation is made by means of the Particle Swarm Optimization (PSO) algorithm and transformations were parameterised with quaternions. To analyse the obtained results, the Monte-Carlo method was used. After thousands of runs of PSO optimization, values of quaternion parts and tensor entries were obtained. Then, thousands of realizations of generally anisotropic tensors described with normal distributions of entries were generated. Each of these tensors was the subject of separate PSO optimization, and the distributions of rotated tensor entries were obtained. The results obtained were compared with solutions of the method based on the Frobenius distances (Danek et al. 2013).
\end{abstract}

Keywords: seismic, anisotropy, elasticity tensor, effective tensor, PSO, entries zeroing method

\section{INTRODUCTION}

The elasticity tensor $c$ relates linearly the stress $\sigma$ and the strain $\varepsilon$ tensors, as shown below in the constitutional equation of Hookean solids (Slawinski 2010):

$\sigma_{i j}=\sum_{k=1}^{3} \sum_{l=1}^{3} c_{i j k l} \varepsilon_{k l} \quad$ for $i, j \in\{1,2,3\}$

It is the general way of the description of elastic properties to provide a more detailed description of the medium than elastic moduli commonly used by engineers and in seismic exploration. As $\mathrm{P}$ - and S-wave propagation is dependent on particular elements of the elasticity tensor, one can estimate the elasticity tensor based on seismic measurements. Laboratory surveys on the rock samples have recently been complemented by in situ researches. The anisotropic elasticity tensor, containing all 21 independent elements, can be estimated based on the results of multiazimuth walkaway vertical seismic profiling (VSP) as described in Dewangan \& Grechka (2003). The essential condition is the sufficient polar and azimuthal coverage of the data. 
Inversion for elasticity coefficients in the most general case requires slowness and polarization vectors for $\mathrm{P}$-waves as well as for $\mathrm{S}_{1}$ - and $\mathrm{S}_{2}$-waves. The receivers are placed inside the borehole, and overburden complexity influences the estimation of the horizontal slowness components at the geophone levels. If these values are poorly estimated, the inversion for $c_{i j k l}$ may not be feasible.

Anisotropic elasticity tensors can usually be approximated with an effective tensor belonging to one of the eight symmetry classes, according to the symmetry planes and axes of the medium. The specific class indicates medium complexity, while the orientation of the planes and axes shows the general direction of layers and fissures. The changes of the medium over time caused by fracturing may also be visible with the analysis of elasticity tensor symmetries and orientations.

\section{METHODS}

\section{Elasticity tensor}

As a consequence of symmetries of the strain and stress tensors, symmetry of elasticity tensor is observed: $c_{i j k l}=c_{j i k l}=c_{k l i j}$ and the number of independent entries in a generally anisotropic case is limited to 21. Equation (1) can be rewritten as follows:

$$
\left[\begin{array}{c}
\sigma_{11} \\
\sigma_{22} \\
\sigma_{33} \\
\sqrt{2} \sigma_{23} \\
\sqrt{2} \sigma_{13} \\
\sqrt{2} \sigma_{12}
\end{array}\right]=\left[\begin{array}{cccccc}
c_{1111} & c_{1122} & c_{1133} & \sqrt{2} c_{1123} & \sqrt{2} c_{1113} & \sqrt{2} c_{1112} \\
c_{1122} & c_{2222} & c_{2233} & \sqrt{2} c_{2223} & \sqrt{2} c_{2213} & \sqrt{2} c_{2212} \\
c_{1133} & c_{2233} & c_{3333} & \sqrt{2} c_{3323} & \sqrt{2} c_{3313} & \sqrt{2} c_{3312} \\
\sqrt{2} c_{1123} & \sqrt{2} c_{2223} & \sqrt{2} c_{3323} & 2 c_{2323} & 2 c_{2313} & 2 c_{2312} \\
\sqrt{2} c_{1113} & \sqrt{2} c_{2213} & \sqrt{2} c_{3313} & 2 c_{2313} & 2 c_{1313} & 2 c_{1312} \\
\sqrt{2} c_{1112} & \sqrt{2} c_{2212} & \sqrt{2} c_{3312} & 2 c_{2312} & 2 c_{1312} & 2 c_{1212}
\end{array}\right]\left[\begin{array}{c}
\varepsilon_{11} \\
\varepsilon_{22} \\
\varepsilon_{11} \\
\sqrt{2} \varepsilon_{23} \\
\sqrt{2} \varepsilon_{13} \\
\sqrt{2} \varepsilon_{12}
\end{array}\right]
$$

Tensor $c$, representing a Hookean solid, belongs to one of eight material symmetry classes, as shown in several works (Forte \& Vianello 1996, Bona et al. 2004). Symmetry classes are characterized by their symmetry groups, which are groups of transformations $g$ (subgroups of 3D rotation group, $\mathrm{SO}(3)$ ) leaving tensor $c$ of given symmetry class invariant:

$c=g(c)$
The simplest class is general anisotropy, while other symmetry classes (described as non-trivial) are the following: monoclinic symmetry, trigonal symmetry, orthotropic symmetry, tetragonal symmetry, transverse isotropy, cubic symmetry, and isotropy. The following symmetry groups correspond to the symmetry classes covered in this paper (Bona et al. 2004):

$G^{\text {orth }}=\left\{ \pm I, \pm M_{e 1}, \pm M_{e 2}, \pm M_{e 3}\right\}$

$G^{\text {tetra }}=\left\{\begin{array}{l} \pm I, \pm R_{\frac{\pi}{2}, e 3}, \pm R_{\frac{-\pi}{2}, e 3}, \pm M_{e 1}, \pm M_{e 2}, \\ \pm M_{e 3}, \pm M_{(1,1,0)}, \pm M_{(1,-1,0)}\end{array}\right\}$

$G^{T I}=\left\{ \pm I, \pm R_{\theta, e 3}, \pm M_{v}\right\}$

for $\theta \in[0,2 \pi), v \in e_{1} e_{2}$ plane

$\mathrm{R}_{\theta, e i}$ describes the rotation by $\theta$ around $e_{i}$, and $M_{v}$ describes the reflection through the plane with normal $v$. Tensor $c$ is of even-rank; therefore, $\pm I$ belongs to each of its symmetry groups, and as a result, if $c$ is invariant under $A$, it is also invariant under $-A$. We can consider only rotation for Equations (4)-(6) without the loss of generality.

A geological example of the transversely isotropic medium is a set of parallel layers in a sedimentary basin. The orthotropic solid is a TI medium with a perpendicular set of parallel fractures. The tetragonal medium is a special case of an orthotropic solid where fractures occur with equal density along both horizontal axa.

Practically, tensors obtained from real seismic measurements always belong to the generally anisotropic symmetry class, since the results are burdened with measurement errors. However, one can approximate tensor $c$ by means of another tensor $c^{\text {sym }}$ belonging to the particular symmetry class. With the fixed orientation of coordinates, in the sense of the Frobenius norm, the best approximation of $c^{\text {sym }}$ is the orthogonal projection of tensor $c$ onto the linear space of all tensors of specific class $\left(L^{\text {sym }}\right)$ (Kochetov \& Slawinski 2009). 
The operator of the projection $-p r_{s y m}(c)$, is given with the following formula:

$c^{s y m}=p r_{s y m}(c)=\int_{G^{s y m}}(g(c)) d \mu(g)$

with the integration over symmetry group $G^{\text {sym }}$ with respect to the invariant $\mu$ normalized to satisfy $\mu\left(G^{\text {sym }}\right)=1$. For classes having finite symmetry groups (all apart from isotropy and transverse isotropy), the integral reduces to a finite sum. Diner et al. (2011) described explicit expressions of $c^{s y m}$ for these symmetries in the coordinate system associated with the symmetry group of each class. For example, an explicit expression for orthotropic symmetry is shown below:

$c^{\text {ortotropic }}=\frac{1}{4}\left(C+M_{e 1}^{T} C M_{e 1}+M_{e 2}^{T} C M_{e 2}+M_{e 3}^{T} C M_{e 3}\right)$

The quality of tensor approximation is evaluated with the squared distance between $c$ and $c^{\text {sym }}$ as shown below:

$d_{s y m}^{2}=\|C\|^{2}-\left\|C^{s y m}\right\|^{2}$

The tensor giving the least value of this squared distance is referred as an effective tensor (Kochetov \& Slawinski 2009). For all symmetry groups except isotropy, the operator of the projection $p r_{s y m}(c)$ as well as obtained distance (9), are dependent on the orientation of the symmetry group.

$$
\tilde{A}(q)=\left[\begin{array}{cccccc}
A_{11}^{2} & A_{12}^{2} & A_{13}^{2} & \sqrt{2} A_{12} A_{13} & \sqrt{2} A_{11} A_{13} & \sqrt{2} A_{11} A_{12} \\
A_{21}^{2} & A_{22}^{2} & A_{23}^{2} & \sqrt{2} A_{22} A_{23} & \sqrt{2} A_{21} A_{23} & \sqrt{2} A_{21} A_{22} \\
A_{31}^{2} & A_{32}^{2} & A_{33}^{2} & \sqrt{2} A_{32} A_{33} & \sqrt{2} A_{31} A_{33} & \sqrt{2} A_{31} A_{32} \\
\sqrt{2} A_{21} A_{31} & \sqrt{2} A_{22} A_{32} & \sqrt{2} A_{23} A_{33} & A_{23} A_{32}+A_{22} A_{33} & A_{23} A_{31}+A_{21} A_{33} & A_{22} A_{31}+A_{21} A_{32} \\
\sqrt{2} A_{11} A_{31} & \sqrt{2} A_{12} A_{32} & \sqrt{2} A_{13} A_{33} & A_{13} A_{32}+A_{12} A_{33} & A_{13} A_{31}+A_{11} A_{33} & A_{12} A_{31}+A_{11} A_{32} \\
\sqrt{2} A_{11} A_{21} & \sqrt{2} A_{12} A_{22} & \sqrt{2} A_{13} A_{23} & A_{13} A_{22}+A_{12} A_{23} & A_{13} A_{21}+A_{11} A_{23} & A_{12} A_{21}+A_{11} A_{22}
\end{array}\right]
$$

introduced by Hamilton (1844), are structures given in the following form:

$q=a+b i+c j+d k$

where: $a$ is real part, vector $(b, c, d)$ is imaginary part and $i, j, k$ are imaginary units satisfying $i^{2}=j^{2}=k^{2}=i j k=-1$. The unitary quaternion $(\|q\|=1)$ can be used to describe rotation in 3D and respective orthogonal matrix is given with (Kochetov \& Slawinski 2009):

$$
A(q)=\left[\begin{array}{ccc}
a^{2}+b^{2}-c^{2}-d^{2} & -2 a d+2 b c & 2 a c+2 b d \\
2 a d+2 b c & a^{2}-b^{2}+c^{2}-d^{2} & -2 a b+2 c d \\
-2 a c+2 b d & 2 a b+2 c d & a^{2}-b^{2}-c^{2}+d^{2}
\end{array}\right]
$$

The rotation is about the axis of components $[b, c, d]$ by angle $\theta=2 \arccos (a)$; thus, opposite quaternions correspond to the same matrix. The rotation $A(q)$ gives rise to an orthogonal transformation of the space of tensor classes $r \rightarrow A(q) r A(q)^{T}$, where $r$ is a symmetric $3 \times 3$ matrix, representing the stress or the strain tensor. Transformation $A(q)$ corresponds to the transformation of tensor $C$ given by: $C \rightarrow \tilde{A} C \tilde{A}^{T}$, where an orthogonal $6 \times 6$ matrix $\tilde{A}$ is given by the following expression (Kochetov \& Slawinski 2009):
To find the effec-

tive tensor without a priori assumption of orientation, one should minimize the squared distance under all orientations, and the tensor obtained in the result is referred to as an absolute effective (Danek et al. 2013). The problem of the minimization of Equation (9) as a function of orientation is nonlinear.

\section{Rotations with quaternions}

In this contribution, we parameterise rotations $\mathrm{SO}(3)$ with quaternions of norm equal 1 to carry out computations. Quaternions, which were 
of orientation described with quaternion $q$ as follows:

$$
\min \Delta_{s y m}^{2}(q):=\|C\|^{2}-\left\|p r_{s y m}\left(C^{e f f}\right)\right\|^{2}
$$

The result of optimization is the value of $q$ giving the absolute minimum of the Frobenius distance. The tensor referred to as $C^{e f f}$ is the absolute effective tensor, expressed in the coordinate system rotated using $A(q)$ matrix. The procedure described by several authors (i.e. Kochetov \& Slawinski 2009, Danek et al. 2013) includes the selection of a particular symmetry class and then the optimization of the target function, which is a Frobenius norm (14), over different orientations of the coordinate system. This results in computational form of Equation (13):

$$
f_{\text {target }}=\Delta_{s y m}^{2}(q):=\sum_{i=1}^{6} \sum_{j=1}^{6}\left(C_{i j}-C_{i j}^{e f f}\right)^{2}
$$

In this contribution, we propose a new approach to the search for the absolute effective tensor. In the entries zeroing method we assume a particular symmetry group and wish to obtain a tensor similar to the effective tensor of this class by means of the rotation of the coordinate system of the measured tensor using $\tilde{A}(q)$. It is a different approach to transformation because the coordinate system of the effective tensor is rotated in the method described above. The target function, specific for each particular symmetry class, describes the similarity of the rotated measured tensor to the form of tensor characteristic for a given class.

Let us analyse the orthotropic symmetry class (described also in Gierlach \& Danek 2017). In this case, the effective tensor $C^{\text {ortotropic }}$ has several entries equal to zero, if expressed in a system where coordinate axes are normal to the symmetry planes:

$$
C^{\text {orthotropic }}=\left[\begin{array}{cccccc}
c_{1111} & c_{1122} & c_{1133} & 0 & 0 & 0 \\
c_{1122} & c_{2222} & c_{2233} & 0 & 0 & 0 \\
c_{1133} & c_{2233} & c_{3333} & 0 & 0 & 0 \\
0 & 0 & 0 & 2 c_{2323} & 0 & 0 \\
0 & 0 & 0 & 0 & 2 c_{1313} & 0 \\
0 & 0 & 0 & 0 & 0 & 2 c_{1212}
\end{array}\right]
$$

One can find explicit formulas for other symmetry classes in Slawinski 2016.
Optimization aims to find rotation matrix $A(q)$ for which the values of respective entries are nearly zeroed. If we describe the measured elasticity tensor as $c$ and particular entries as $c_{i j k l}$ (see Equation (2)), the target function can be written as follows:

$$
f_{\text {target }}=\sum_{i=1}^{3} \sum_{k=1}^{2} \sum_{l=k+1}^{3} c_{i k k l}^{2}+\sum_{i=1}^{2} \sum_{l=2}^{i+1} c_{i 31 l}^{2}
$$

In other words, the target function is the sum of these 12 independent entries of a tensor squared (out of 21 independent entries of generally anisotropic tensor) which are located neither on the diagonal nor in first quarter of the tensor (column 1-3 and row 1-3). After the optimization is completed, we obtain a quaternion suggesting the orientation of the coordinate system whose axes are normal to the symmetry planes. The absolute effective tensor is the original tensor rotated with the obtained rotation matrix and with respective entries set to zero (those which were optimized to be zeroed).

One can easily modify the target function according to the characteristic patterns of the tensor in order to use this method for other symmetry classes. For a tetragonal tensor, we have to take into account following conditions:

$c_{1111}=c_{2222}, c_{1133}=c_{2233}$ and $c_{2323}=c_{1313}$

For this symmetry class, we add to the target function (16) squared differences of entries which are expected to be equal. Conditions for transverse isotropy also contain equality:

$2 c_{1212}=c_{1111}-c_{1122}$

The target function for the TI symmetry class is given with the sum of Formula (16) and the differences of left-hand and right-hand sides of all equations in (17) and (18). One should remember that the obtained orientation is one of an infinite number of possibilities; therefore, as in this case, one of the coordinate axes is parallel to the axis of symmetry and another two axes are arbitrary.

The method described in this contribution can also be applied for the cubic symmetry class. In 
this case, the target function also needs to consider the following equations:

$c_{1111}=c_{2222}=c_{3333}, c_{1133}=c_{1122}=c_{2233}$

and $c_{2323}=c_{1212}=c_{1313}$

Effective isotropic tensor entries are calculated with explicit formulas, and the values of particular entries are constant for all orientations of an orthonormal coordinate system. In this case, searching for the orientation of the effective tensor system would obviously be pointless. The method of zeroing entries can also be used for monoclinic and trigonal symmetry classes after some obvious modifications of the target functions.

The problem of searching for the absolute effective tensor is nonlinear. In our contribution, the solution is found with a particle-swarm optimization method (PSO), proposed by Kennedy \& Eberhart (1995). The method is a powerful global optimization technique which can be applied to the wide range of optimization problems without necessity of its internal parameter tuning (see Poli et al. 2007). This algorithm is often used to find the effective tensor (see Danek et al. 2013, Kozubal 2016). PSO is a stochastic method simulating the behaviour of animals searching for a food. Particular members of the swarm are described with the particles placed in the $n$-dimensional solution space. Each particle knows its position (values of solution, in this case four parts of quaternion) and evaluates the target function in its current location. The movement of a single particle through the search space is determined by combining its own current $\left(x_{i}\right)$ and the personal best location $\left(p_{i}\right)$ with the global best location $(g)$ and some random perturbation. The swarm can be additionally subdivided and then $g$ refers to the best location achieved by a member of the group (in this work we treat a swarm as a fully connected graph). New locations are chosen by adding velocity $v_{i}$ to the coordinate $x_{i}$ (it can be treated as a step-size). The next iteration is calculated after all particles are moved. The initial velocity $v_{i}$ is zero, but before first use it is updated.

The algorithm of canonical PSO optimization is presented in Figure 1 as a pseudo-code (based on Poli et al. 2007).

The update of the particle's velocity and position use vectors $\vec{U}\left(0, \Phi_{i}\right)$, which are vectors of random numbers uniformly distributed between $\left[0, \Phi_{i}\right]$, which are randomly generated at each iteration and for each particle. The operator $\otimes$ means the component-wise multiplication. The calculated velocity has to be within the range $\left[-v_{\max }, v_{\max }\right]$. The velocity is usually limited to the highest acceptable value of $x_{i}$ (see Eberhart \& Shi 2000). $\Phi_{1}$ and $\Phi_{2}$ are constriction coefficients limiting particle movements related to the best individual and global positions, respectively.

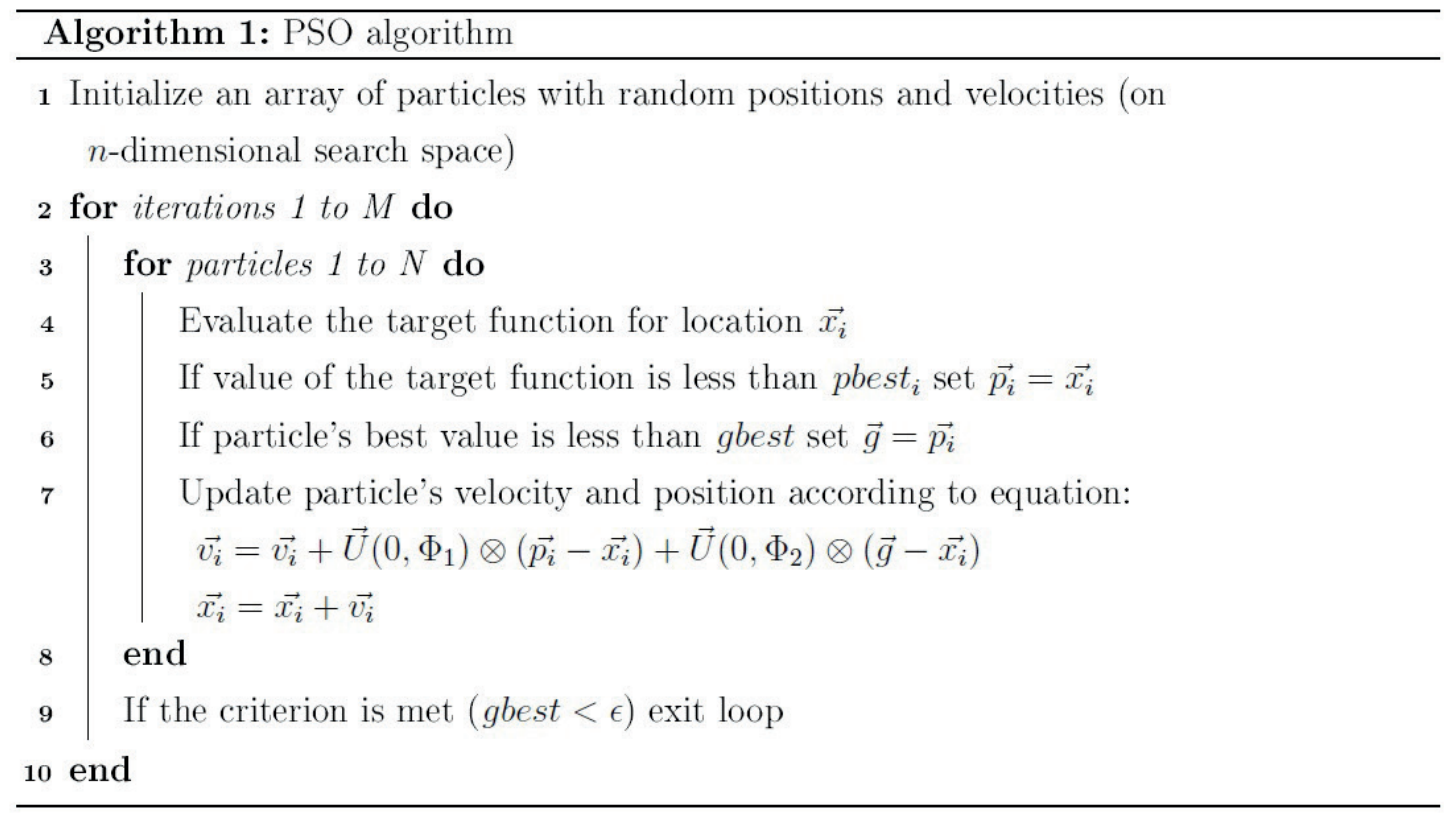

Fig. 1. Simplified algorithm of Particle Swarm Optimization 
In our implementation, we used commonly used values of $\Phi_{i}=2$. The algorithm finishes either when the maximum number of iterations or when sufficiently good fit is achieved.

A single value of the target function arises as a result of the rotation of the coordinate system with a given quaternion and the calculation of a specific sum according to the particular symmetry class. Since we use only unit quaternions to parameterise the rotations, we have to normalize the tested quaternions in each iteration.

\section{RESULTS}

All analyses in this paper are conducted using the generally anisotropic elasticity tensor (20) obtained by Dewangan \& Grechka (2003). The source of 21 elastic stiffness coefficients $c_{i j}$ was a multiazimuth, multicomponent VSP data set acquired in New Mexico. The entries are density-scaled so their unit is square kilometre per square second $\left[\mathrm{km}^{2} / \mathrm{s}^{2}\right]$ :

$C=\left[\begin{array}{cccccc}7.8195 & 3.4495 & 2.5667 & \sqrt{2}(0.1374) & \sqrt{2}(0.0558) & \sqrt{2}(0.1239) \\ 3.4495 & 8.1295 & 2.3589 & \sqrt{2}(0.0812) & \sqrt{2}(0.0735) & \sqrt{2}(0.1692) \\ 2.5667 & 2.3589 & 7.0908 & \sqrt{2}(-0.0092) & \sqrt{2}(0.0286) & \sqrt{2}(0.1655) \\ \sqrt{2}(0.1374) & \sqrt{2}(0.0812) & \sqrt{2}(-0.0092) & 2(1.6636) & 2(-0.0787) & 2(0.1053) \\ \sqrt{2}(0.0558) & \sqrt{2}(0.0735) & \sqrt{2}(0.0286) & 2(-0.0787) & 2(2.0660) & 2(-0.1517) \\ \sqrt{2}(0.1239) & \sqrt{2}(0.1692) & \sqrt{2}(0.1655) & 2(0.1053) & 2(-0.1517) & 2(2.4270)\end{array}\right]$

The values are obtained as a result of the measurement and inversion of slowness and polarization vectors for the elasticity tensor. Thus, one should treat the tensor as a set of normal distributions of entries, not just their strict values, because the entries are burdened with measurement errors. The matrix of standard deviations of tensor entries is given below (Danek \& Slawinski 2015):

$$
S= \pm\left[\begin{array}{cccccc}
0.1656 & 0.1122 & 0.1216 & \sqrt{2}(0.1176) & \sqrt{2}(0.0774) & \sqrt{2}(0.0741) \\
0.1122 & 0.1862 & 0.1551 & \sqrt{2}(0.0797) & \sqrt{2}(0.1137) & \sqrt{2}(0.0832) \\
0.1216 & 0.1551 & 0.1439 & \sqrt{2}(0.0856) & \sqrt{2}(0.0662) & \sqrt{2}(0.1010) \\
\sqrt{2}(0.1176) & \sqrt{2}(0.0797) & \sqrt{2}(0.0856) & 2(0.0714) & 2(0.0496) & 2(0.0542) \\
\sqrt{2}(0.0774)) & \sqrt{2}(0.1137) & \sqrt{2}(0.0662) & 2(0.0496) & 2(0.0626) & 2(0.0621) \\
\sqrt{2}(0.0741) & \sqrt{2}(0.0832) & \sqrt{2}(0.1010) & 2(0.0542) & 2(0.0621) & 2(0.0802)
\end{array}\right]
$$

The first tests of our method were conducted using the single tensor (20). The procedure described in the previous section was applied 1000 times in order to obtain the orthotropic effective tensor. Each run produced one quaternion, and, since the optimization algorithm is based on a random search of the solution space, the obtained results differ. However, different quaternions may describe the same rotation of a tensor expressed in several coordinate systems and gives an equal target function, so there are several global minima defining optimal solution for effective tensor (see Equations (23) and (25)). A histogram of the target function values (Fig. 2A) shows that most of the results are close to the global minimum but there are several values diverging from the minimum. It was decided arbitrarily that values right of the vertical line ( $>0.301)$ constituting $2.7 \%$ of the total number of values should be eliminated from the solutions set. The single optimization (one run of PSO procedure) did not bring a satisfying result and so repeated optimization had to be applied. The stopping criterion for the procedure is defined as follows: if three identical (to the numerical accuracy) quaternions are found, one of them is accepted as a solution. The histogram of values obtained with this approach, which is shown in the right plot of Figure 2, is narrower and all the target function values are less than 0.301 .

In the crossplots of particular parts of quaternions obtained with single optimization (Fig. 3), one can see that values are concentrated around certain points.

Circles are black or grey, depending on the target function value. It is clearly visible that in case of some points we do not observe values of target function lower than 0.301 . It suggests that these points represent local minima.

For example, by running our procedure, we obtained the following quaternion:

$$
q=-0.00636+0.81548 i+0.57795 j-0.03050 k
$$


A

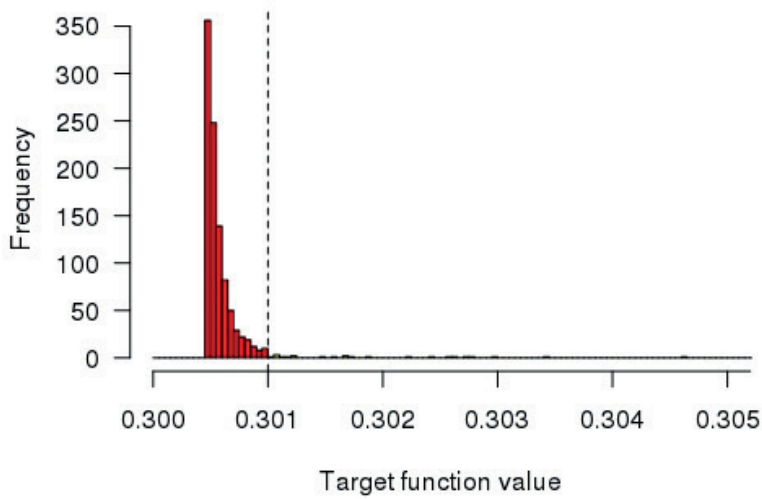

$\mathrm{B}$

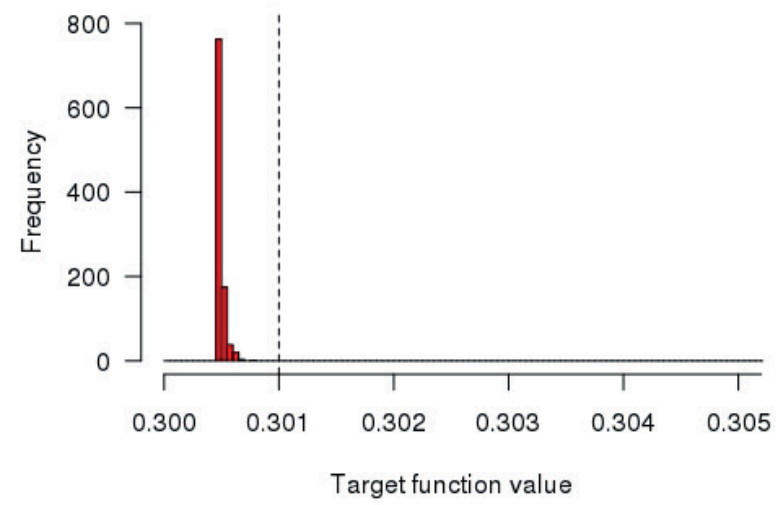

Fig. 2. Histograms of the final values of the target function. The described procedure was performed 1000 times to produce the orthotropic effective tensor: A) with a single optimization; $B$ ) with the optimization repeated to eliminate diverging values

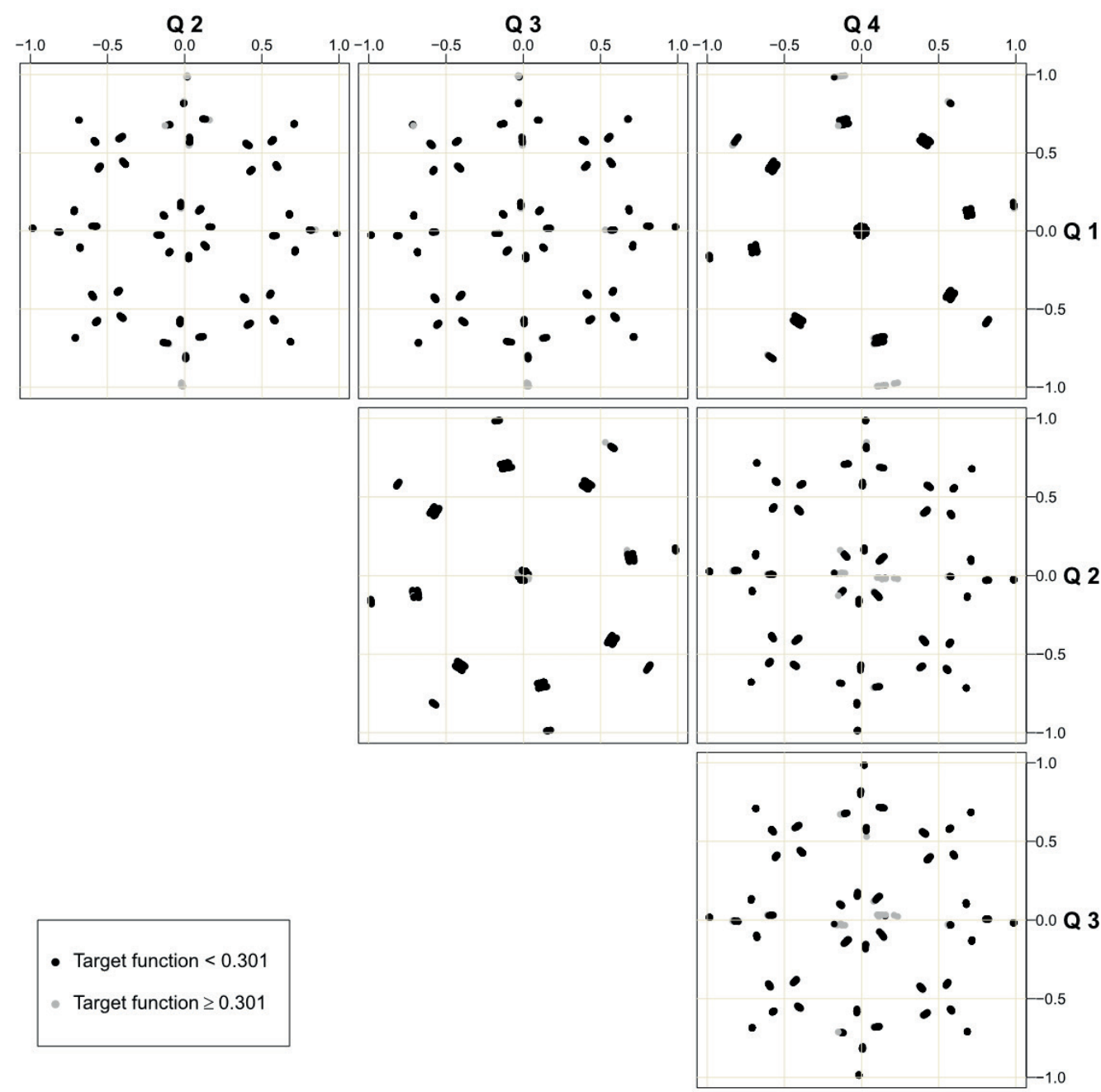

Fig. 3. Solutions of the procedure for orthotropic class with single optimization (1000 runs). Scatterplot matrix contains all the pairwise crossplots of the particular quaternion parts (Q1-Q4). The tone of points depends on the value of the target function for the solution 
The target function for this rotation is equal to 0.30046 , and the tensor expressed in transformed coordinate system is as follows:
For the non-zero entries (grey bars), one can clearly see that each entry has three possible values.

Solutions di$C=\left[\begin{array}{cccccc}8.3776 & 3.3626 & 2.4888 & \sqrt{2}(0.1136) & \sqrt{2}(-0.0203) & \sqrt{2}(0.1545) \\ 3.3626 & 7.7742 & 2.4267 & \sqrt{2}(-0.0691) & \sqrt{2}(-0.0799) & \sqrt{2}(-0.0244) \\ 2.4888 & 2.4267 & 7.0810 & \sqrt{2}(-0.0389) & \sqrt{2}(-0.0404) & \sqrt{2}(0.2068) \\ \sqrt{2}(0.1136) & \sqrt{2}(-0.0691) & \sqrt{2}(-0.0389) & 2(2.0781) & 2(0.0715) & 2(0.1479) \\ \sqrt{2}(-0.0203) & \sqrt{2}(-0.0799) & \sqrt{2}(-0.0404) & 2(0.0715) & 2(1.6500) & 2(-0.0267) \\ \sqrt{2}(0.1545) & \sqrt{2}(-0.0244) & \sqrt{2}(0.2068) & 2(0.1479) & 2(-0.0267) & 2(2.3315)\end{array}\right]$

vided into three groups, depending on the value of $c_{11}$ entry, show easily noticeable patterns in the crossplots of part values (Fig. 5).

The same value of target function was obtained with different quaternion (24) giving tensor (25) as a result of rotation:

$q=0.01708-0.98520 i+0.16850 j-0.02621 k$

$c_{11}$ was used to distinguish 3 forms in which resulting tensor is observed (compare Fig. 4) If the values of quaternions are recalculated to Euler angles, one can also notice the clear division of the points (Fig. 6). The number of point groups in the quaternion crossplot is doubled, compared to Euler angle crossplots, $C=\left[\begin{array}{cccccc}7.7744 & 3.3622 & 2.4262 & \sqrt{2}(0.0800) & \sqrt{2}(-0.0690) & \sqrt{2}(0.0248) \\ 3.3622 & 8.3783 & 2.4893 & \sqrt{2}(0.0201) & \sqrt{2}(01142) & \sqrt{2}(-0.1541) \\ 2.4262 & 2.4893 & 7.0809 & \sqrt{2}(0.0404) & \sqrt{2}(-0.0390) & \sqrt{2}(-0.2068) \\ \sqrt{2}(0.0800) & \sqrt{2}(0.0201) & \sqrt{2}(0.0404) & 2(1.6502) & 2(-0.0720) & 2(-0.0264) \\ \sqrt{2}(-0.0690) & \sqrt{2}(01142) & \sqrt{2}(-0.0390) & 2(-0.0720) & 2(2.0779) & 2(-0.1477) \\ \sqrt{2}(0.0248) & \sqrt{2}(-0.1541) & \sqrt{2}(-0.2068) & 2(-0.0264) & 2(-0.1477) & 2(2.3311)\end{array}\right]$ as two opposite quaternions describe the same set of angles. Solutions divided with respect to other non-zero entries also give

Please note, that this is the same tensor describing the same medium but in a different coordinate system.

All of the accepted results for many different quaternions (1000) are shown in Figure 4. Please note that when a tensor is expressed in a different coordinate system, its entries can have different values. Nevertheless, this is still exactly the same tensor corresponding with the optimized target function. For entries that were to be zeroed according to the form of the orthotropic tensor (black bars), the distributions of values are approximately symmetrical with the central part about zero. However, none of these entries is equal to zero for any tensor. Additionally, in this example, the measurement errors of the original tensor were not applied in the optimization process. It is also visible that values can be divided into several groups, because the distributions are not continuous. clear patterns but sets of point groups are not always the same.

Since the tensor entries should be treated as distributions, further analyses were conducted with a statistical approach. This means that tensors being subject of inversion and rotation were realizations of normal distributions having mean values given with Equation (20) and standard deviations given with Equation (21) for particular tensor entries.

Three specific tensors from among those 50,000 obtained were additionally marked with a circle, square, or triangle in Figure 7. It confirms the earlier observation about the swapping of some entries for tensors in different coordinate systems (e.g. $c_{11}$, $c_{22}, c_{33}$ or $c_{12}, c_{13}, c_{23}$. The tensors were chosen such that each one is contained in one of three separate groups of solutions shown in Figures 5 and 6 . While for non-zero entries pattern of values is clearly visible, we cannot see any rule in zeroed entries. 


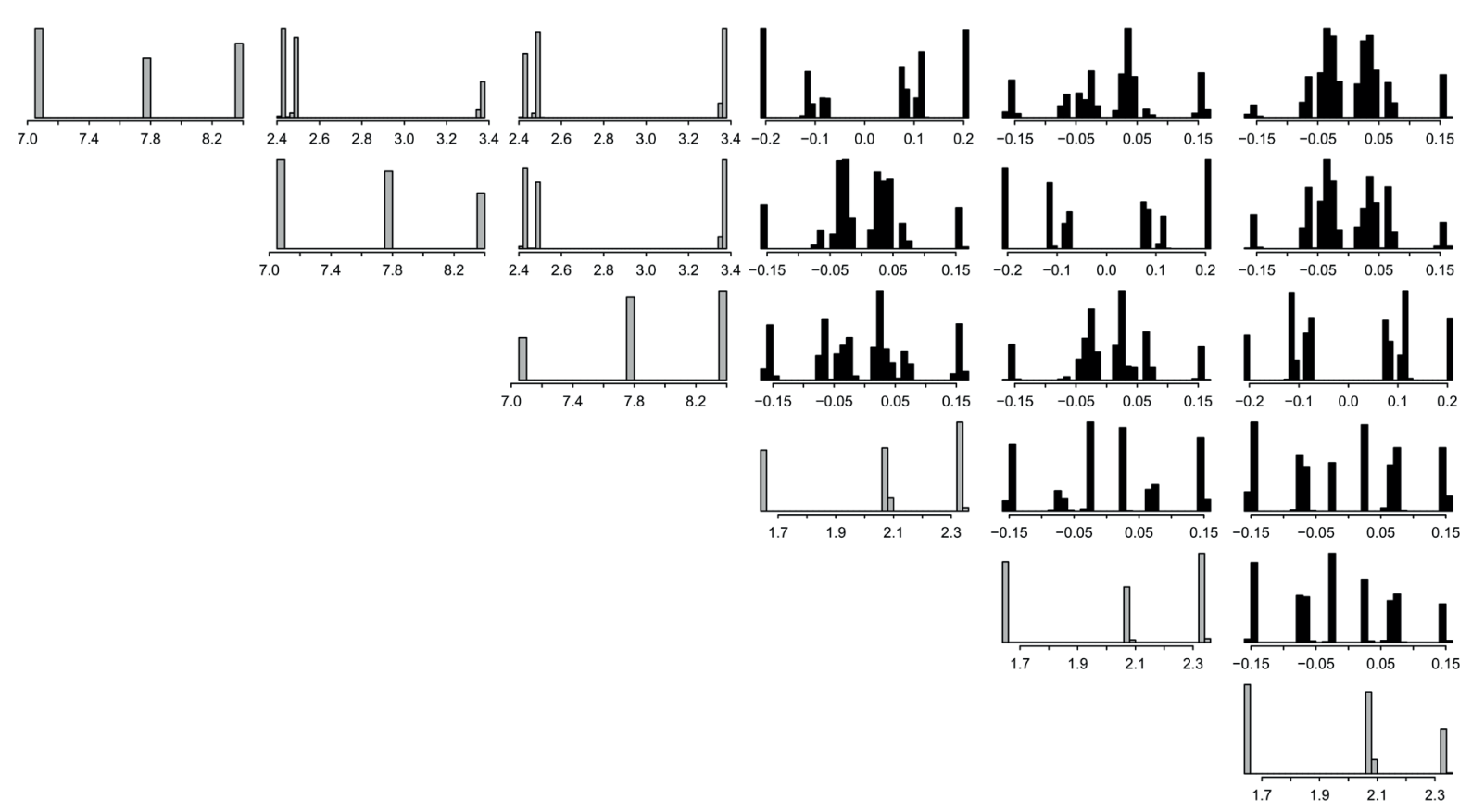

Fig. 4. Distribution matrix of the tensor entries. Values obtained with the described procedure for one specific orthotropic tensor. Plots represent respective entries in the $6 \times 6$ matrix (black bars - zeroed entries, grey bars - non-zero entries)

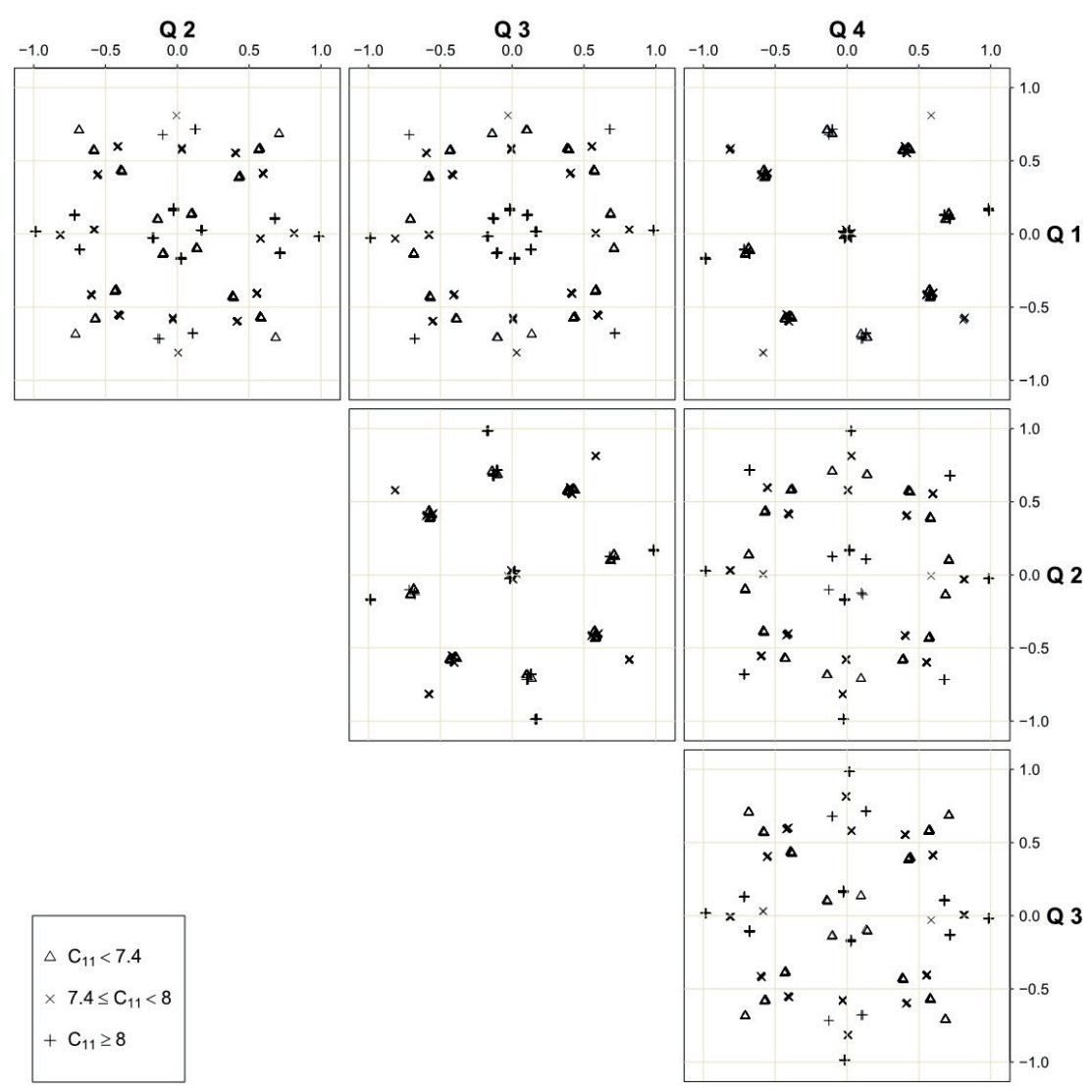

Fig. 5. Scatterplot matrix containing values of particular parts of quaternions for orthotropic class. Points are divided into three groups depending on the value of $c_{11}$ entry obtained from rotation with specific quaternion 

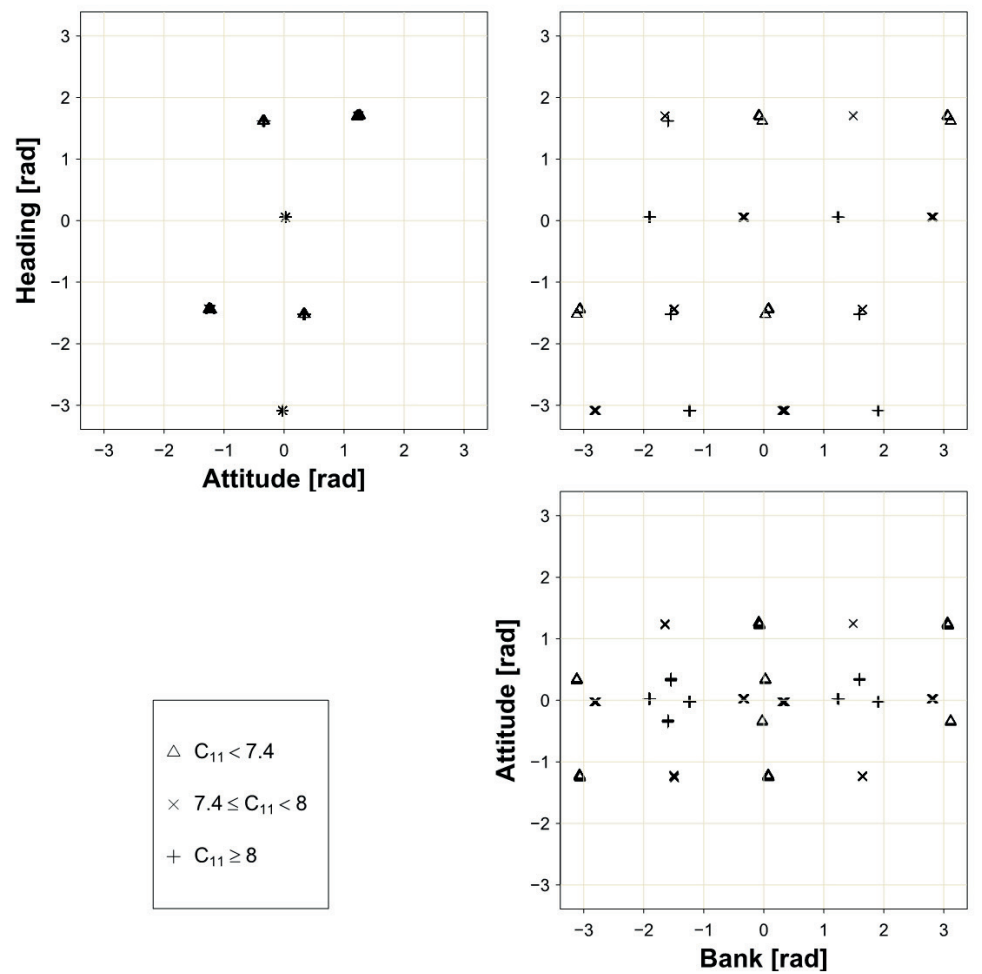

Fig. 6. Scatterplot matrix containing values of Euler angles for orthotropic class. Points are divided into three groups depending on the value of $c_{11}$ entry obtained through rotation with specific set of angles
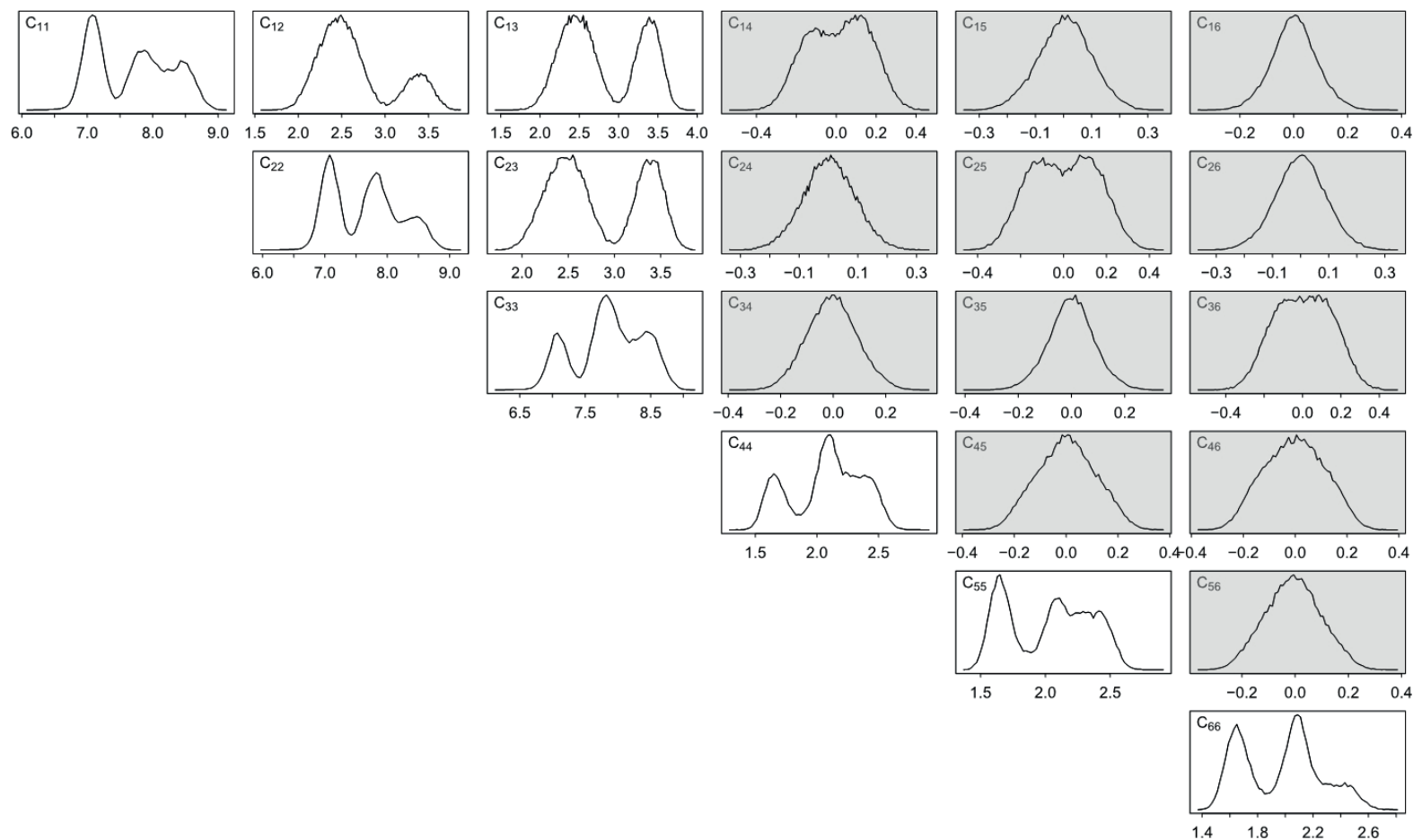

Fig. 7. Distribution matrix of the tensor entries for the orthotropic class. 50,000 realizations of tensor generated randomly with normal distributions (instead of exact values of entries) were rotated with quaternions obtained as results of separate optimizations. Entries of three chosen tensors are marked respectively with a circle, triangle and square 
The next analyses were conducted for the tetragonal symmetry class. In the distributions of the entries obtained as a result of the procedure with the target function specific for tetragonal symmetry (Fig. 8), which were repeated 1000 times, one can notice that the zeroing of respective entries was not as effective as for the orthotropic class. This is visible especially for $c_{45}, c_{46}$ and $c_{56}$ entries, where the obtained values are at quite a large distance from zero. The non-zero entries do not show that characteristic pattern known from the orthotropic class. The pairs of entries expected to be equal have very similar distributions (in respective pairs) and comprise two peaks, each one constituting about half of the values. The other entries have unimodal pseudonormal distributions (differences less than 0.01). The values of obtained quaternions (Fig. 9) comprise fewer point groups than for the orthotropic class. One group is omitted, because it does not satisfy the criteria to be treated as a global minimum. Here, we can also see the equal partition of quaternions with respect to the $c_{11}$ entry value. In the crossplots of Euler angles calculated from mentioned quaternions (Fig. 10), one can see an even more distinct partition for the two groups. There are only eight sets of angles giving a minimum of the target function.

The distributions obtained as a result of a separate procedure for 50,000 randomly generated tensors (Fig. 11) confirm what we expected after the analysis of the distributions for one specific tensor. Even though we operate with normal distributions (not exact values of entries), there is nearly a zero probability to have $c_{45}$ entry zeroed. Some entries are apparently unimodal, but it is an effect of the merging of two normal distributions with means differing about $0.1\left(c_{44}, c_{55}, c_{66}\right.$ - compare Figs. 8 and 11). While entries expected to be equal appear to have nearly identical distributions, we cannot say that entries expected to be zeroed can be equal to zero.

The same analysis conducted for the procedure with the target function specified for the transverse isotropic symmetry class shows very similar results (Fig. 12), since a TI class is the subgroup of tetragonal class. The $c_{45}$ value cannot be zeroed while different conditions describing the TI class seems to be satisfied. The non-zero entries all seems to be unimodal or bimodal with nearly equal modes.
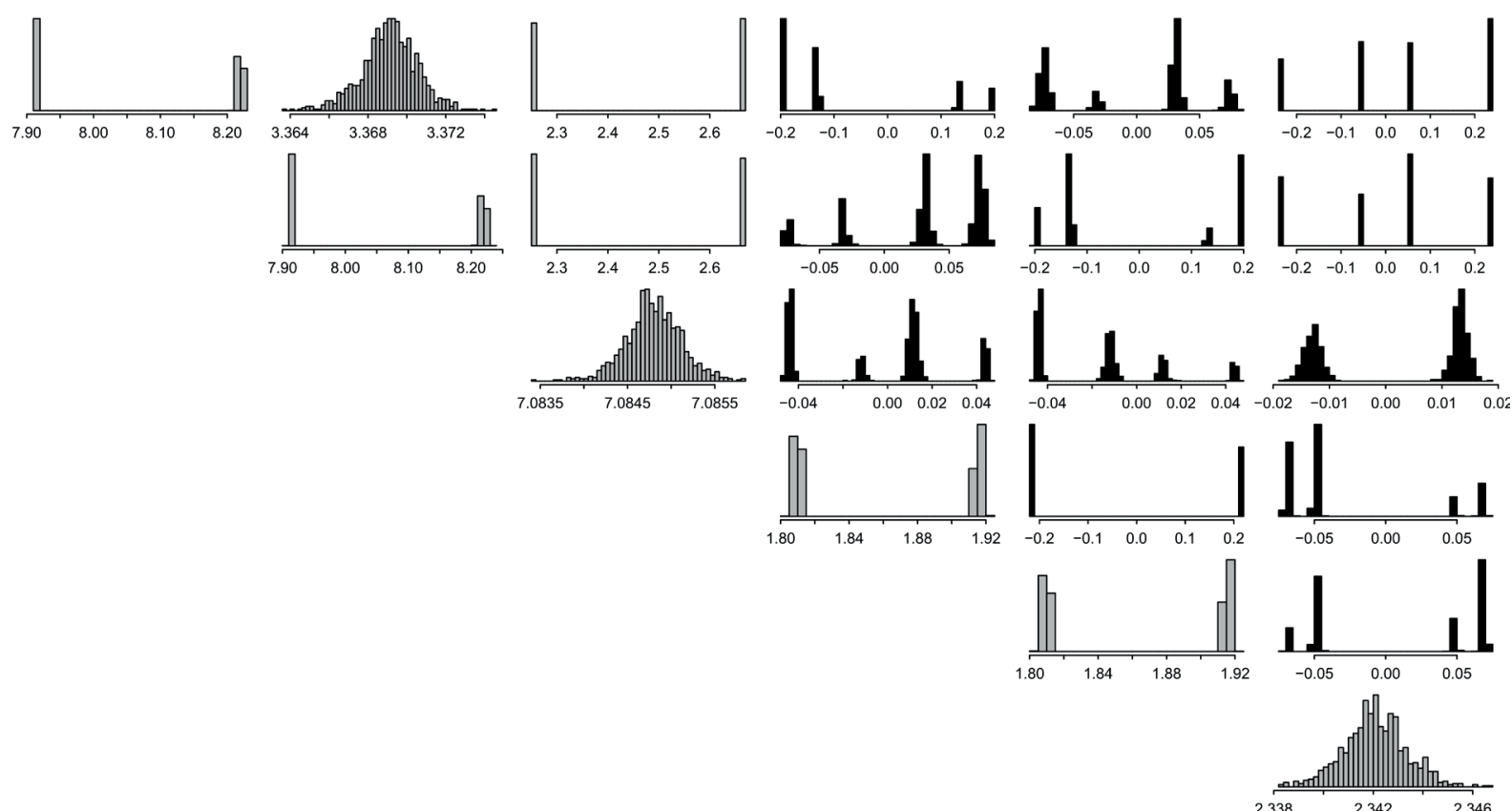

Fig. 8. Distribution matrix of the tensor entries. One specific tensor was rotated with quaternions obtained with 1000 runs of procedure for tetragonal symmetry class (black bars - zeroed, grey bars - non-zero entries) 


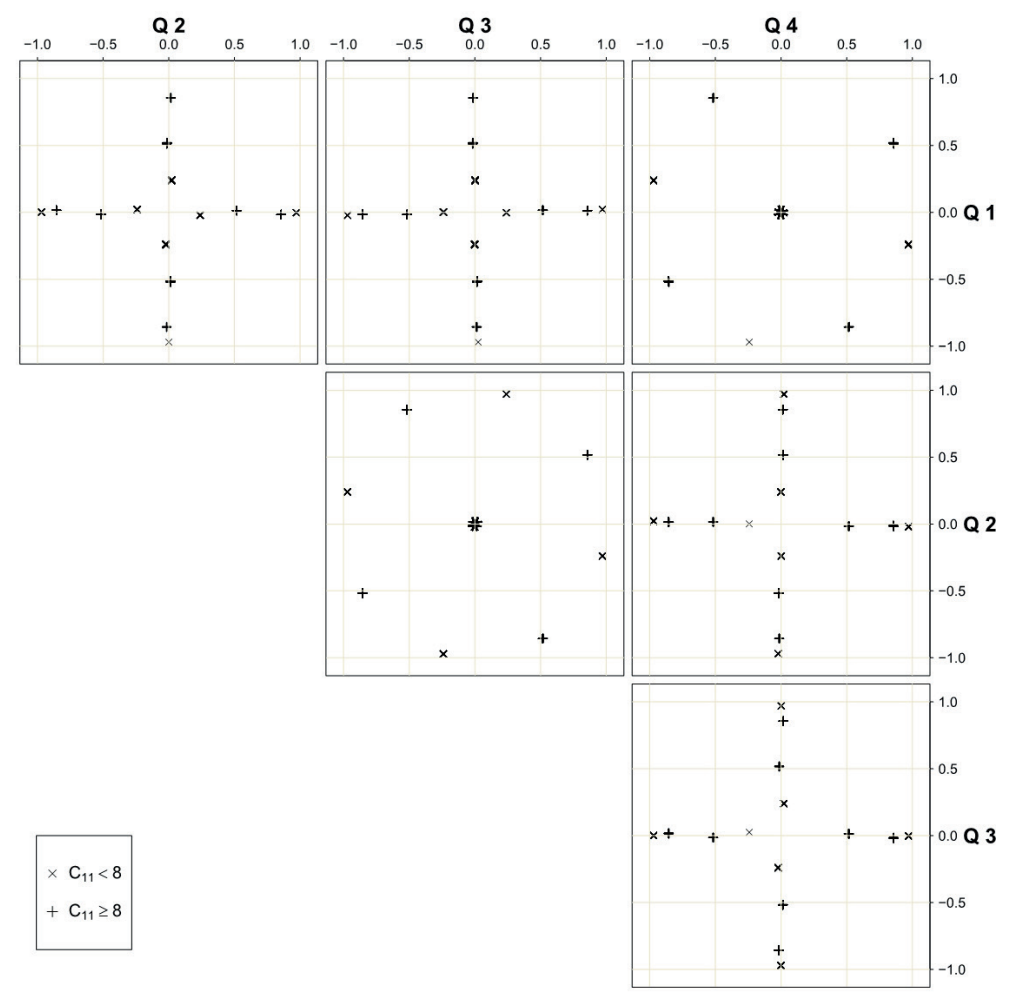

Fig. 9. Scatterplot matrix containing values of particular parts of quaternions for tetragonal class. Points are divided into three groups depending on the value of the entry obtained from rotation with a specific quaternion
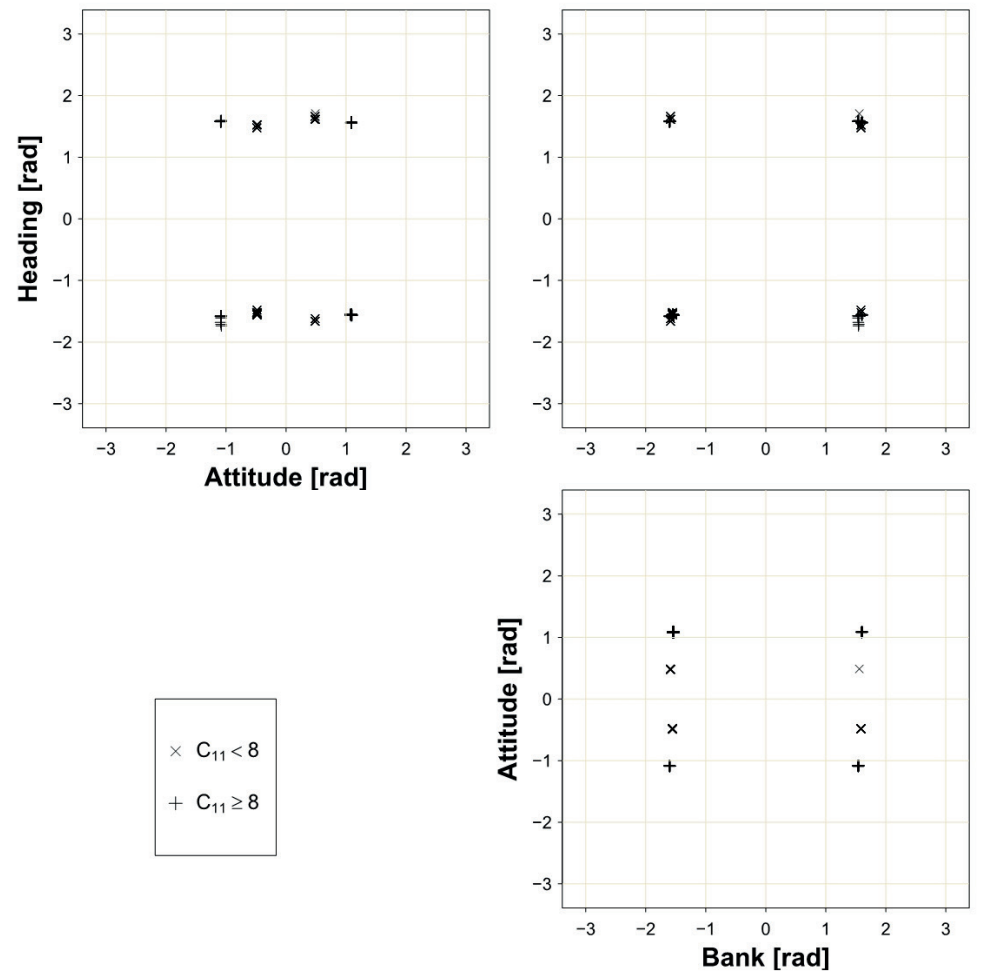

Fig. 10. Scatterplot matrix containing values of Euler angles for tetragonal class. Points are divided into three groups depending on the value of $c_{11}$ entry obtained through rotation with specific set of angles 

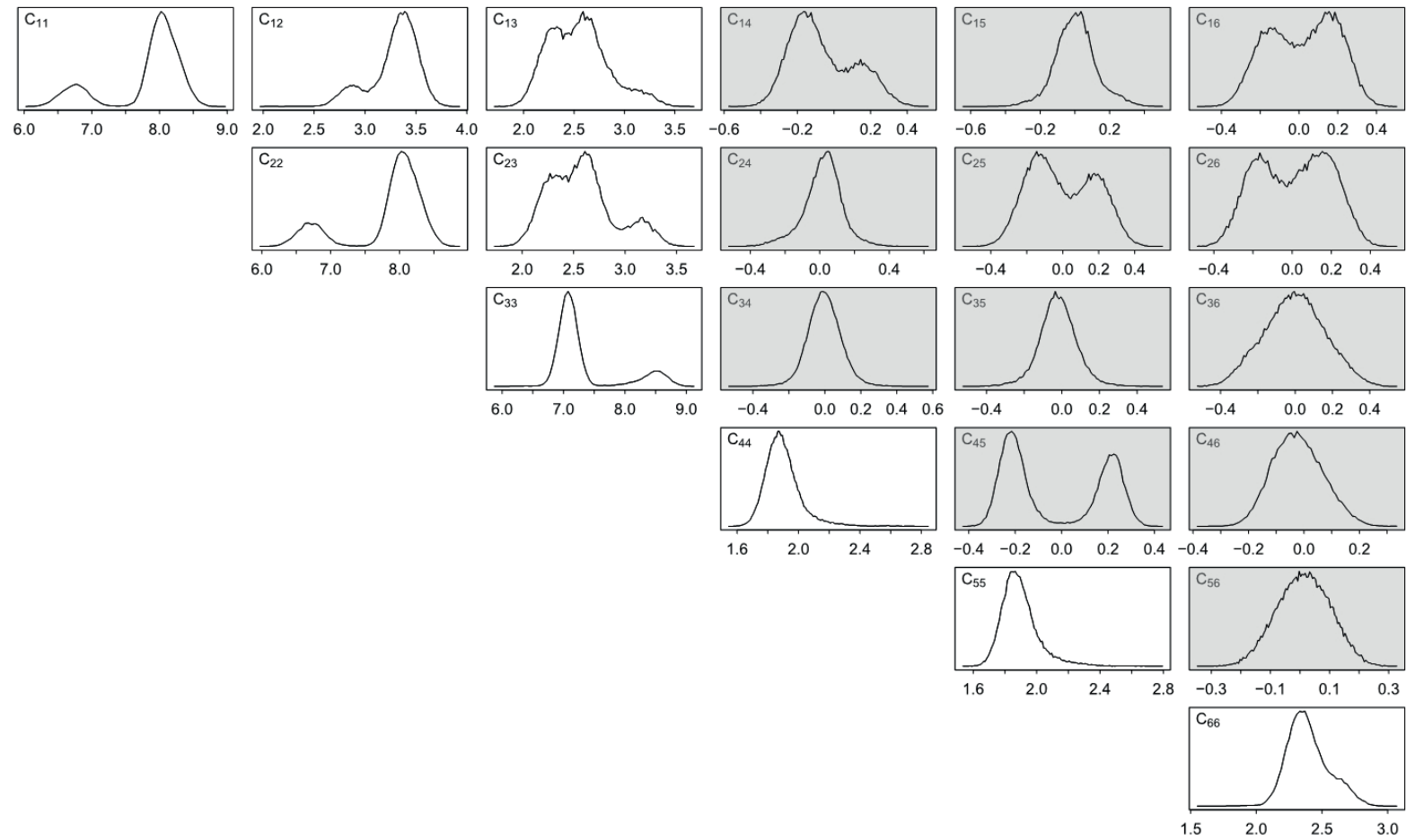

Fig. 11. Distribution matrix of the tensor entries for the tetragonal class. 50,000 realizations of tensor generated randomly with normal distributions were rotated with quaternions obtained as results of separate optimizations
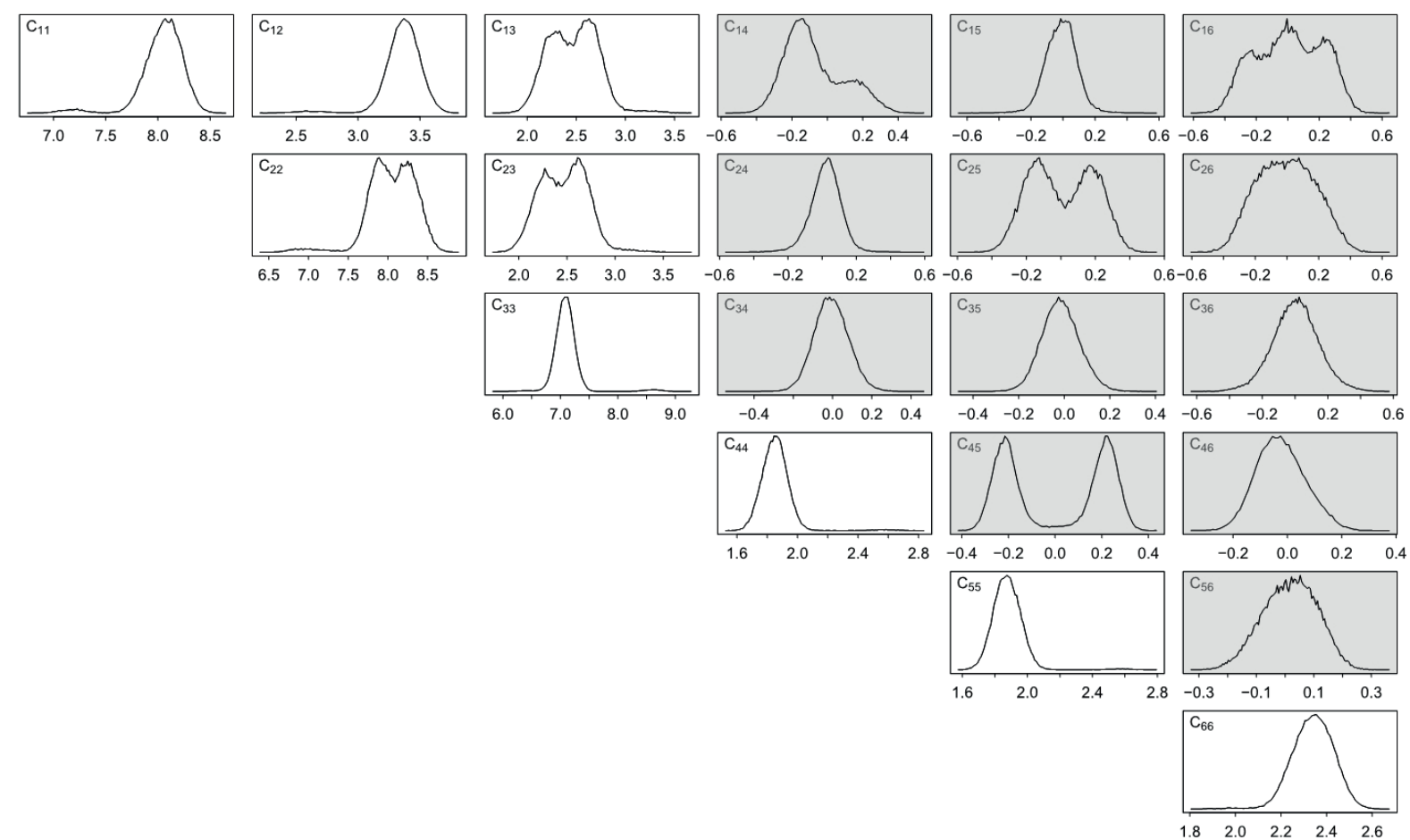

Fig. 12. Distribution matrix of the tensor entries for the transverse isotropic class. 50,000 realizations of tensor generated randomly with normal distributions were rotated with quaternions obtained as results of separate optimizations 


\section{DISCUSSION AND CONCLUSIONS}

The described entries zeroing method is a tool to obtain an effective tensor of a certain symmetry from measured generally anisotropic tensor. In this contribution, the orthotropic, tetragonal, and transverse isotropy classes were analysed. Obviously, applying the proposed method for other classes is trivial and require only target function modifications similar to the ones used in presented examples. Comparison of the obtained results strongly suggests that this tensor belongs to the orthotropic symmetry class. This conclusion is supported by the fact that entry $c_{45}$ cannot be zeroed for tetragonal and TI symmetry class (compare Figs. 11 and 12). Please note, that in error-free case original tensor cannot be considered as an orthotropic (see Fig. 4).

In this work, an algorithm was tested for three symmetry classes. The choice of the classes was based on a priori information about the medium being measured in the original field experiment. As the isotropic effective tensor is calculated analytically and is independent of the orientation, the described method does not apply to this class.

For the orthotropic class, there are the same possible values of the respective entries (i.e. $c_{11}$, $c_{22}, c_{33}$ ), but recorded with a different frequency. The interpretation of this phenomenon is that the same tensor is expressed in three different coordinate systems rotated by $\pi / 2$ (please see Fig. 6 and compare Danek et al. 2013).

The obtained results can be compared with the effects of previous methods based on the optimization of the Frobenius distance. A comparison of the respective entries of the effective tensor belonging to a given class can be performed instantaneously. When comparing the orientations described with the quaternion or set of Euler angles, one should remember that in distance-based methods the coordinate system of the effective tensor is rotated to the coordinate system of the measured tensor, while in the entries zeroing method the transformation is done in reverse direction.

In general, the obtained results are in agreement with those obtained by Danek et al. (2013). In all analysed cases, very similar solutions can be found for both methods. In some cases, e.g. tetragonal class, the entry zeroing method is not sensitive enough to provide all expected solutions (only two possible values of the bank angle with $\pi$ step, while Danek et al. have found eight possible orientations with $\pi / 4$ step). Nevertheless, we consider this result satisfactory.

The proposed method may be used to replace or complement the existing method of describing the symmetry class and orientation of the medium based on the elasticity tensor measured in situ with vertical seismic profiling, with its less complex target function and process of optimization being an advantage of the described method.

This research was partially supported by the National Science Centre of Poland under contract No. DEC-2013/11/B/ST10/0472 and by AGH University of Science and Technology, Faculty of Geology, Geophysics and Environmental Protection as a part of the statutory project No. 11.11.140.613.

\section{REFERENCES}

Bona A., Bucataru I. \& Slawinski M.A., 2004. Characterization of Elasticity-Tensor Symmetries Using SU(2). Journal of Elasticity, 75, 3, 267-289.

Danek T., Kochetov M. \& Slawinski M.A., 2013. Uncertainty analysis of effective elasticity tensors using quaternion-based global optimization and Monte-Carlo method. The Quarterly Journal of Mechanics and Applied Mathematics, 66, 2, 253-272.

Danek T. \& Slawinski M.A., 2015. On choosing effective elasticity tensors using a Monte-Carlo Method. Acta Geophysica, 63, 1, 45-61.

Dewangan P. \& Grechka V., 2003. Inversion of multicomponent, multiazimuth, walkaway VSP data for the stiffness tensor. Geophysics, 68, 3, 1022-1031.

Diner C., Kochetov M. \& Slawinski M.A., 2011. On choosing effective symmetry classes for elasticity tensors. The Quarterly Journal of Mechanics and Applied Mathematics, 64, 1, 57-74.

Eberhart R.C. \& Shi Y., 2000. Comparing inertia weights and constriction factors in particle swarm optimization. [in:] Proceedings of the 2000 Congress on Evolutionary Computation: CEC00: July 16-19, 2000, La Jolla Marriott Hotel, La Jolla, California, USA, IEEE Service Center, 84-88.

Forte S. \& Vianello M., 1996. Symmetry Classes for Elasticity Tensor. Journal of Elasticity, 43, 2, 81-108.

Gierlach B. \& Danek T., 2017. Obtaining orthotropic elasticity tensor using entries zeroing method. [in:] $E G U$ European Geosciences Union: general assembly 2017: Vienna, Austria, 23-28 April 2017, Geophysical Research Abstracts, 19, [on-line:] http://meetingorganizer.copernicus.org/EGU2017/EGU2017-438.pdf [access: 10.06.2018] 
Hamilton W.R., 1844. On a new species of imaginary quantities connected with a theory of quaternions. Proceedings of the Royal Irish Academy, 2, 424-434.

Kennedy J. \& Eberhart R.C., 1995. Particle swarm optimization. [in:] 1995 IEEE International Conference on Neural Networks: proceedings, the University of Western Australia, Perth, Western Australia, 27 November-1 December 1995, IEEE, 1942-1948.

Kochetov M. \& Slawinski M.A., 2009. On obtaining effective orthotropic elasticity tensors. The Quarterly Journal of Mechanics and Applied Mathematics, 62, 2, 149-166.

Kozubal A., 2016. Global and local stochastic optimization in effective elasticity tensors evaluation. [in:] $15^{\text {th }}$
International Conference on Geoinformatics - Theoretical and Applied Aspects (Geoinformatics 2016): Proceedings of a meeting held 10-13 May 2016, Kiev, Ukraine, EAGE, 454

Poli R., Kennedy J. \& Blackwell T., 2007. Particle swarm optimization - An overview. Swarm Intelligence, 1, 1, 33-57.

Shoemake K., 1985. Animating rotation with quaternion curves. ACM SIGGRAPH computer graphics, 19, 3, 245-254.

Slawinski M.A., 2010. Waves and Rays in Elastic Continua. World Scientific, Singapore.

Slawinski M.A., 2016. Wavefronts and rays in seismology. Answers to unasked questions. World Scientific, Singapore. 